Internat. J. Math. \& Math. Sci.

Vol. 24, No. 8 (2000) 529-532

S0161171200003902

(C) Hindawi Publishing Corp.

\title{
RECURSIVE DETERMINATION OF THE ENUMERATOR FOR SUMS OF THREE SQUARES
}

\author{
JOHN A. EWELL
}

(Received 28 July 1999 and in revised form 12 September 1999)

\begin{abstract}
For each nonnegative integer $n, r_{3}(n)$ denotes the number of representations of $n$ by sums of three squares. Here presented is a two-step recursive scheme for computing $r_{3}(n), n \geq 0$.
\end{abstract}

Keywords and phrases. Representations of numbers by sums of three squares, combinatorial identities.

2000 Mathematics Subject Classification. Primary 11E25; Secondary 05A20.

1. Introduction. In order to lend greater precision to statements of results and methods of proof we begin our discussion with a definition.

Definition 1.1. As usual, $\mathbb{P}:=\{1,2,3, \ldots\}, \mathbb{N}:=\mathbb{P} \cup\{0\}$ and $\mathbb{Z}:=\{0, \pm 1, \pm 2, \ldots\}$. Then, for each $n \in \mathbb{N}$,

$$
r_{3}(n):=\left|\left\{(h, j, k) \in \mathbb{Z}^{3} \mid n=h^{2}+j^{2}+k^{2}\right\}\right| ;
$$

and $q_{0}(n):=$ the number of partitions of $n$ into distinct odd parts. The function $q_{0}(n), n \in \mathbb{N}$, is generated by the infinite product expansion

$$
\prod_{1}^{\infty}\left(1+x^{2 n-1}\right)=\sum_{0}^{\infty} q_{0}(n) x^{n}
$$

which is valid for each complex number $x$ such that $|x|<1$.

The outstanding result about $r_{3}(n), n \in \mathbb{N}$, was first presented by Legendre. We state his result in the following theorem.

THEOREM 1.2. If $S:=\left\{n \in \mathbb{P} \mid n=4^{k}(8 m+7)\right.$, for some $\left.k, m \in \mathbb{N}\right\}$, then for each $n \in S, r_{3}(n)=0$; and, for each $n \in \mathbb{N}-S, r_{3}(n)>0$.

Based on his theory of ternary quadratic forms, Gauss gave the first complete proof of this theorem in his now famous book Disquistiones Arithmaticae.

In this note, our major objective is to give a two-step recursive determination of the sequence $r_{3}(n), n \in \mathbb{N}$. This is accomplished by the following two results. 
THEOREM 1.3. For each $n \in \mathbb{N}$,

$$
\sum_{k=0}(-1)^{k(k+1) / 2} q_{0}\left(n-\frac{k(k+1)}{2}\right)= \begin{cases}(-1)^{m}, & \text { if } n=m(3 m \pm 1), \\ 0, & \text { otherwise. }\end{cases}
$$

Here, summation ranges over all $k \in \mathbb{N}$ such that $n-k(k+1) / 2 \geq 0$.

THEOREM 1.4. For each $n \in \mathbb{N}$,

$$
\begin{aligned}
r_{3}(n)= & q_{0}(n)-\sum_{k=1}(-1)^{k(3 k-1) / 2} q_{0}\left(n-\frac{k(3 k-1)}{2}\right)(6 k-1) \\
& +\sum_{k=1}(-1)^{k(3 k+1) / 2} q_{0}\left(n-\frac{k(3 k+1)}{2}\right)(6 k+1) .
\end{aligned}
$$

As before, summation extends as far as the arguments of $q_{0}$ remain nonnegative.

For a proof of Theorem 1.3, see [1, pages 1-2]. Section 2 is dedicated to proof of Theorem 1.4.

2. Proof of Theorem 1.4. Our proof is based on the following two identities, each of which is valid for each complex number $x$ such that $|x|<1$.

$$
\begin{aligned}
\prod_{1}^{\infty}\left(1-x^{n}\right)\left(1-x^{2 n-1}\right) & =\sum_{-\infty}^{\infty}(-1)^{n} x^{n^{2}}, \\
\prod_{1}^{\infty}\left(1-x^{n}\right)^{3}\left(1-x^{2 n-1}\right)^{2} & =\sum_{-\infty}^{\infty}(6 n+1) x^{n(3 n+1) / 2} .
\end{aligned}
$$

Identity (2.1) is due to Gauss; and, elementary proofs of it abound, cf. [3, page 284]. The second identity (2.2) is due to Basil Gordon [2, page 285]. In passing we observe that the cube of the right-hand side of (2.1) generates the sequence $(-1)^{n} r_{3}(n)$, $n \in \mathbb{N}$. Hence, we multiply both sides of $(2.2)$ by $\prod_{n=1}^{\infty}\left(1-x^{2 n-1}\right)$ to get

$$
\begin{aligned}
\sum_{0}^{\infty}(-1)^{n} r_{3}(n) x^{n} & =\prod_{1}^{\infty}\left(1-x^{n}\right)^{3}\left(1-x^{2 n-1}\right)^{3} \\
& =\prod_{1}^{\infty}\left(1-x^{2 n-1}\right)\left\{1-\sum_{k=1}^{\infty}(6 k-1) x^{k(3 k-1) / 2}+\sum_{k=1}^{\infty}(6 k+1) x^{k(3 k+1) / 2}\right\} \\
& =\sum_{j=0}^{\infty}(-1)^{j} q_{0}(j) x^{j}\left\{1-\sum_{k=1}^{\infty}(6 k-1) x^{k(3 k-1) / 2}+\sum_{k=1}^{\infty}(6 k+1) x^{k(3 k+1) / 2}\right\}
\end{aligned}
$$

Now, we expand the product of the two series, and subsequently equate coefficients of like powers of $x$ to prove our theorem.

Our recursive two-step algorithm proceeds as follows:

(i) use the recursive determination of $q_{0}$ in Theorem 1.3 to compile a table of values of $q_{0}$, as in Table 2.1,

(ii) in terms of these computed values of $q_{0}$ we then utilize Theorem 1.4 to compile a table of values of $r_{3}$, as in Table 2.2. 
TABLE 2.1.

\begin{tabular}{|cc||cc|}
\hline \hline$n$ & $q_{0}(n)$ & $n$ & $q_{0}(n)$ \\
\hline \hline 0 & 1 & 13 & 3 \\
1 & 1 & 14 & 3 \\
2 & 0 & 15 & 4 \\
3 & 1 & 16 & 5 \\
4 & 1 & 17 & 5 \\
5 & 1 & 18 & 5 \\
6 & 1 & 19 & 6 \\
7 & 1 & 20 & 7 \\
8 & 2 & 21 & 8 \\
9 & 2 & 22 & 8 \\
10 & 2 & 23 & 9 \\
11 & 2 & 24 & 11 \\
12 & 3 & 25 & 12 \\
\hline
\end{tabular}

TABLE 2.2

\begin{tabular}{|cc||cc|}
\hline \hline$n$ & $r_{3}(n)$ & $n$ & $r_{3}(n)$ \\
\hline \hline 0 & 1 & 13 & 24 \\
1 & 6 & 14 & 48 \\
2 & 12 & 15 & 0 \\
3 & 8 & 16 & 6 \\
4 & 6 & 17 & 48 \\
5 & 24 & 18 & 36 \\
6 & 24 & 19 & 24 \\
7 & 0 & 20 & 24 \\
8 & 12 & 21 & 48 \\
9 & 30 & 22 & 24 \\
10 & 24 & 23 & 0 \\
11 & 24 & 24 & 24 \\
12 & 8 & 25 & 30 \\
\hline
\end{tabular}

CONCluding Remarks. The brief Tables 2.1 and 2.2 are compiled to show the effectiveness of our procedure. In terms of machine computation we observe that for a fixed but arbitrary choice of $n \in \mathbb{P}$, each of Theorems 1.3 and 1.4 requires $0\left(n^{3 / 2}\right)$ running time. Legendre's Theorem 1.2 would provide an excellent check on the accuracy of computation.

For given $n \in \mathbb{P}$, there are formulas which express $r_{3}(n)$ in terms of certain divisor functions; and, also in terms of Jacobi symbols. However, factorization of arguments 
of the divisor functions and denominators of Jacobi symbols is required before these expressions can be utilized. By comparison our procedure is entirely additive in character. In a word, no factorization is required.

ACKNOWLEDGEMENT. The author would like to thank the referee for suggestions leading to improved exposition.

\section{REFERENCES}

[1] J. A. Ewell, Recurrences for two restricted partition functions, Fibonacci Quart. 18 (1980), no. 1, 1-2. MR 81f:10021. Zbl 425.10013.

[2] B. Gordon, Some identities in combinatorial analysis, Quart. J. Math. Oxford Ser. (2) 12 (1961), 285-290. MR 25\#21. Zbl 107.25101.

[3] G. H. Hardy and E. M. Wright, An Introduction to the Theory of Numbers, Clarendon Press, Oxford, 1960, 4th ed. Zbl 086.25803.

John A. EWELL: Department of MATHEMATICAL SCIENCES, NORTHERn ILLINOIS UNIVERSiTy, DEKALB, IL 60115, USA

E-mail address: ewe11@math.niu.edu 


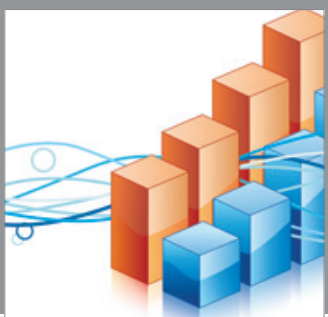

Advances in

Operations Research

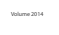

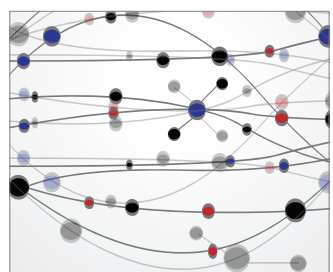

\section{The Scientific} World Journal
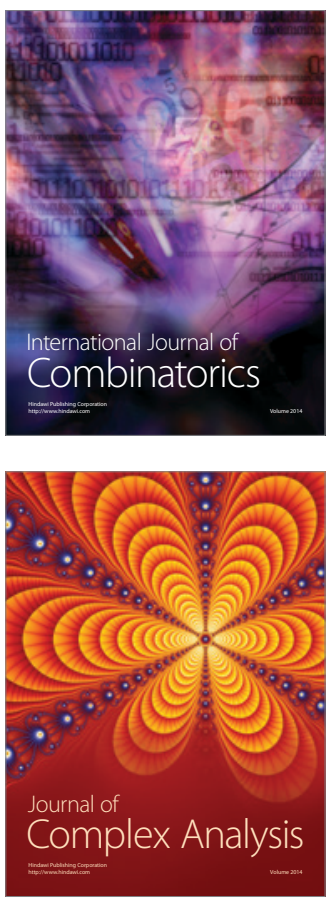

International Journal of

Mathematics and

Mathematical

Sciences
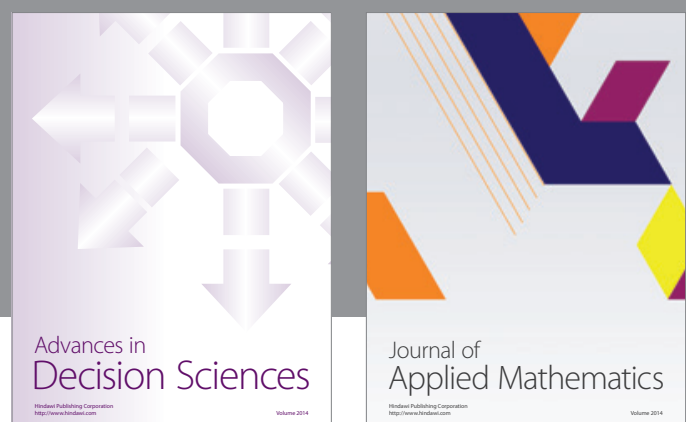

Journal of

Applied Mathematics
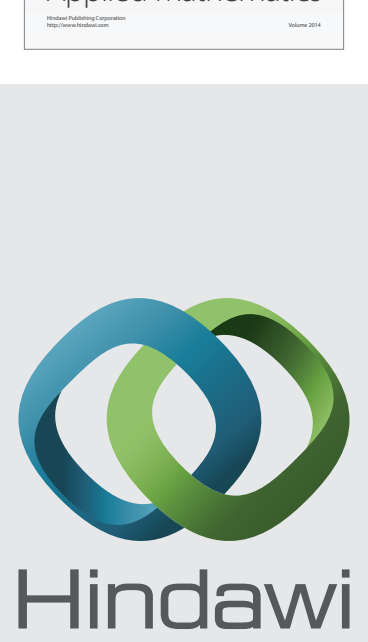

Submit your manuscripts at http://www.hindawi.com
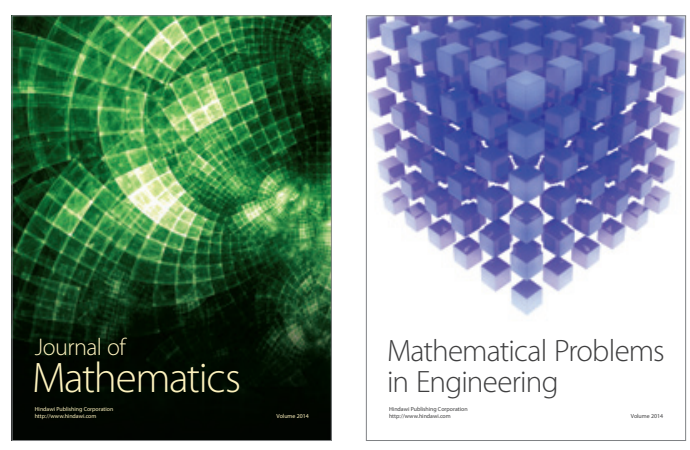

Mathematical Problems in Engineering
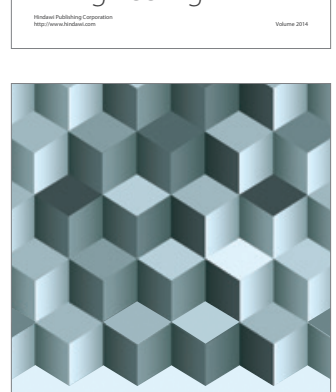

Journal of

Function Spaces
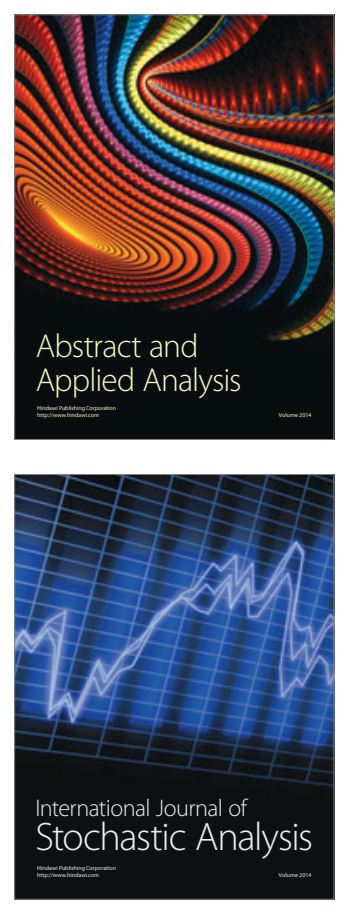

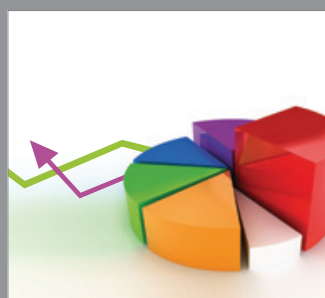

ournal of

Probability and Statistics

Promensencen
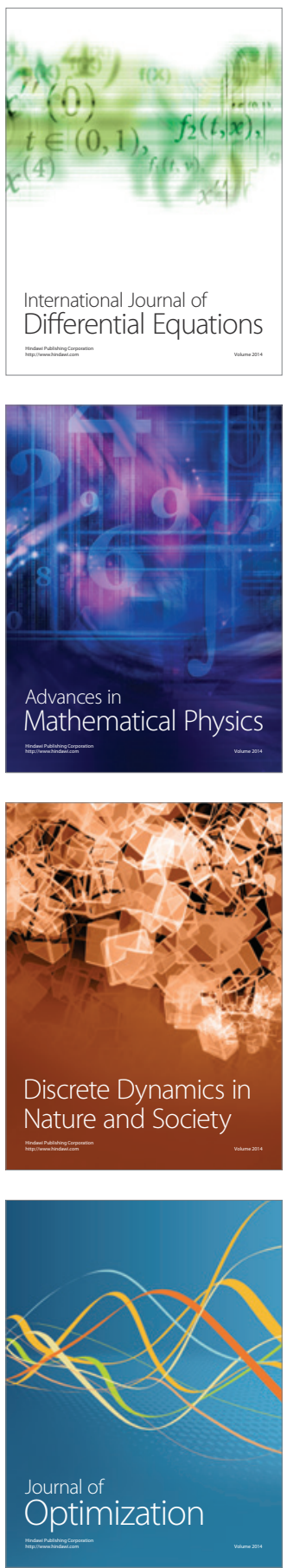